\title{
Constructing a real/virtual archive of architectural material using ubiquitous computing
}

\author{
Norio TOGIYA ${ }^{1}$, Akira BABA ${ }^{2}$ \\ ${ }^{1}$ Graduate School of Interdisciplinary Information Studies, the University of Tokyo \\ ${ }^{2}$ Interfaculty Initiative in Information Studies, the University of Tokyo
}

\begin{abstract}
In recent years, various kinds of cultural material have been digitized. Along with such movements, it is necessary to establish a coexisting relationship between real and virtual archives. In this study, we tried to create a link between real and virtual archives of architectural material using ubiquitous computing.
\end{abstract}

\section{KEYWORDS}

Ubiquitous computing, digital archive, RFID, barcode

\section{Introduction}

Digitization of cultural resources, called digital archive, is currently evolving. We think operating these virtual archives and at the same time maintaining real archive storing actual materials and resources is important. Although the two archives are closely related, their practical operations are often separate. However, it seems to be necessary for them to discharge supplemental duties in order to operate more efficiently. Thus, a system linking the real archive and the corresponding virtual archive is required. We decided to use ubiquitous computing as a method to link these archive.

Recently, ubiquitous computing has been applied to various kinds of storage facilities for cultural resources.Currently, these applications are focused on certain areas, such as management of museum exhibits or library book stocks. Based on this situation, we interlinked real and virtual archive by using ubiquitous computing. The present project was based on previous research conducted by the authors and others. [1]

\section{Definition of ubiquitous computing and construction of the archive}

\subsection{Ubiquitous computing in archive}

The relationship between real and virtual archive in facilities storing cultural resources is shown in Fig. 1. The left side is the virtual archive and the right side

Received February 17, 2006; Revised March 27, 2006; Accepted April 3, 2006. 1)zi9n-tgy@asahi-net.or.jp, 2) baba@iii.u-tokyo.ac.jp DOI: $10.2201 / \mathrm{NiiPi} .2006 .3 .5$

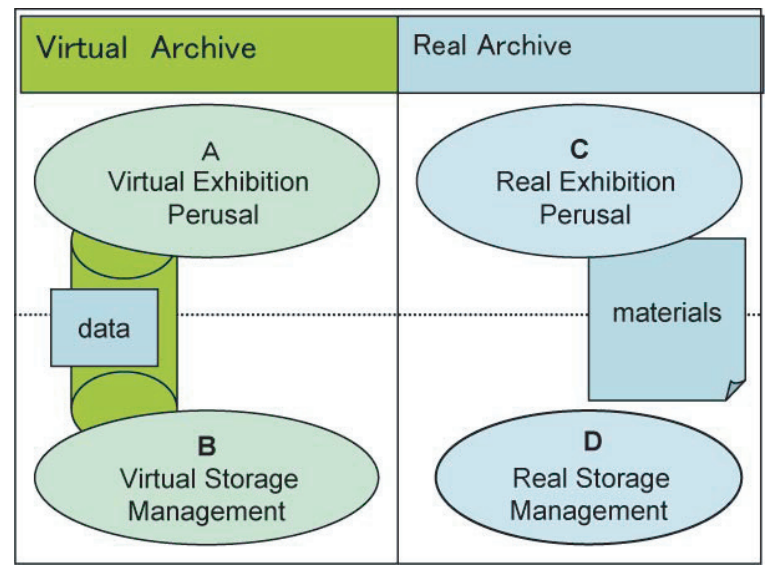

Fig. 1 Relationship between real archive and virtual archive.

is the real archive. The virtual archive is an archive for digitized materials, and the real archive is the repository for the actual materials. The upper part of the figure indicates the front end for exhibition and perusing, while the lower part of the figure indicates the back end for constructing, managing, and storing material. The relationship between the front and back ends can generally be divided into the following four components:
(A) virtual exhibition/perusal,
(B) virtual storage/management,
(C) real exhibition/perusal, and
(D) real storage/management. 
Ideally, these four components commonly share information owned by the respective parts. For instance, catalog data provided in real archive (C) can be used as metadata in the virtual archive of (A). It is also possible to select actual exhibits in (C) by accessing data in the virtual archive of (A). The information for managing the real cultural resources in (D) can be used as notes for digitizing the real archive of (B). Furthermore, the analysis results of digitized materials of (B) that are analyzed with computers can be used for management and research activities of the real archive of (D). As mentioned above, it is possible to promote storage and use of cultural resources more efficiently by sharing information stored in these four components. Ubiquitous computing is considered to be an effective measure for sharing information stored in these four parts.

In this research, ubiquitous computing is defined as "a system distributing anywhere and supporting the various kinds of activities conducted by human beings". There are several examples of applying ubiquitous computing in facilities storing cultural resources. These examples are generally focused on the areas of (C) and (D) at present. For instance, there are exhibition [2] and learning assistance [3], [4] using RFID and PDA in museums for (C) above. For (D), use of the archive as a tool for comprehensive management of collections in a museum has also been studied. In the same way, plans and practices to use RFID for managing collections in libraries have been executed. [5]

In this research, we comprehend the purposes of both the real and the virtual archives from a global perspective, based on the results of the above-mentioned activities. We conducted experiments for using ubiquitous computing mainly in the areas of (A), (B), and (D). The reasons we concentrate on the areas (A), (B), and (D) are mainly that there are few examples of applications in these areas, and (C) perusal/exhibition facilities for real archives are not available in our archive. Thus, materials are generally perused and used with the virtual archive.

Furthermore, we decided to use materials not only in the three areas of (A), (B), and (D) independently, but also to use them to link the three areas in accordance with the construction of the actual virtual archive.

The construction of the virtual archive will follow three processes: selection of resources in (D), digitization in $(\mathrm{C})$, and construction and opening of the virtual archive in (A). Since the virtual archive can be updated at any time, a cycle that returns to (D) again and then moves to (D), (B), and then (A) will be repeated. It is important to use the required data in the respective phases constantly by using ubiquitous computing. Thus, we constructed a ubiquitous computing system that can be used in the respective phases for construct- ing the virtual archive in this project.

\subsection{Construction of the archive}

The comprehensive concept of the real/virtual archive are described in the previous section. Based on the concept, we will explain the verification experiment in which the above-mentioned schemes are partially introduced.

We began conducting the Tsuboi family digital archive project in 2004. In this project, the documents of the Tsuboi family, which have been examined by researchers in the natural science field during and after the modern ages, were sorted and then digitized. [6] One of the construction goals of this project was for both a real and a virtual archive to coexist. In the future, we aim to construct a mutually supplemental archive by using ubiquitous computing in the archive of all of the documents/materials of the Tsuboi family. When we began the project, we needed to conduct experiments to determine how we could apply ubiquitous computing technologies to the project. Thus, we decided to conduct verification experiments in small units before designing and planning the entire project.

The archive we used is an architectural archive of collected documents with regard to the cultural houses constructed by Tsuboi Seitaro [7] in the latter half of the Taisho era. The architectural archive is a repository for documents and materials related to architecture, such as design documents and the relevant documents of the architecture, construction materials, and miniature models of complete houses. One of the significant features of the architectural archive is the fact that its collections take a variety of forms. Thus, the archive has a similar character to that of a museum handling various kinds of solid articles. Management of materials with different shapes is one of the issues that must be addressed for digitizing this kind of archive.

The materials of Tsuboi Residence Architectural Material Archive are extremely diversified, including design drawings, construction materials, images, photos, and project management documents. These materials are stored in several different places with other materials. Since so many kinds of materials are stored, taking out and confirming each of them takes time. For the construction materials in particular, the heights of the materials are about $2 \mathrm{~m}$, and confirming them is very difficult. In the current archive, the storage location for these materials does not have the space for exhibiting them and allowing the public to peruse them. Thus, many of the materials may be perused in the virtual archive or other facilities. We, therefore, decided to construct the real/virtual archive experimentally by using ubiquitous computing for the following purposes. 
The item numbers correspond to the actual use of ubiquitous computing described in Fig. 1.

$<1>$ For entire archive

1) Total amount and contents of the respective materials in the archive can be made comprehensible by assigning ID numbers to all of the materials.

2) For information that will be updated frequently, such as lending status and progress of digitization, the latest data can be referenced on a real-time basis. 3) Status of the real archive can be monitored from the virtual archive, while the status of the virtual archive can be monitored from the real archive.

$<2>$ Functions of archive in the respective archive areas $<2-1>$ Perusal in (A) virtual archive

The digitized materials can be perused and storing/lending status of the materials and storing locations of the materials can be confirmed in the virtual archive.

$<2-2>$ Construction and updates of (B) virtual archive

To construct the virtual archive or increase the contents of the virtual archive by digitalizing the materials, the relevant management, such as completion, incompletion, and progress confirmation of the digitization, can be executed.

$<2-3>$ Storage and management of (D) real archive

1) The lending status, conditions, and storing locations of materials can be confirmed on site.

2) To confirm a large number of materials, a machine can confirm them more efficiently and accurately by checking the assigned codes than by visual confirmation.

3) For a dark stockroom in which actions are very limited, the work load can be reduced by using a lightweight terminal that is more convenient than a personal computer or printed catalog.

$<3>$ Use in construction of virtual archive

The Tsuboi Residence Architectural Material Archive is also constructed through phases in the construction process from (D), then to (B), and finally to (A). Thus, the materials and information will be referenced with a small-sized terminal so that the materials required for photographs can be picked up efficiently in phase (D). In the following phase (B), several points, such as whether the photographed materials are available and whether the contents are the same as in the materials containers, will be confirmed with the small-sized terminal. Then, in phase (A), the status of the real archive of (A) and progress of digitization of (B) can be checked at any time. In the respective phases in various kinds of environments as mentioned above, identical information can be obtained. Thereby, an efficient archive can be constructed.

For the above-mentioned purposes of use, we de- cided to conduct a verification experiment of the real/virtual archive using ubiquitous computing.

\section{Introduction of ubiquitous network into Tsuboi Residence Architectural Material Archive}

\section{1 esign of ubiquitous network}

To construct the real/virtual archive, the project team decided to construct a ubiquitous computing system. The team selected devices and systems that have at least the functions of reading tags such as RFID and IDs recorded on it, calling data corresponding to the tags from the server, and displaying the results on the terminal as their basic components.

Among several devices with the above-mentioned functions, the project team decided to use the UCPhone and its system, [8] developed by YRP Ubiquitous Networking Laboratory. [9] UC-Phone communicates with the server through PHS circuits, and applications required for the server are also attached. The device also has a function for reading barcodes and RFID tags. The project team used this device as the ubiquitous terminal for the project. RFIDs issued by the Ubiquitous ID Center [10] were used as the RFID tags. Codes, the so-called ucode previously provided by the Ubiquitous ID Center, were already set on the RFID tags. The reading functions for barcodes as well as RFID were also added to the terminal. In addition to the ucode-specific barcode, a scheme experimentally expanding the existing JAN codes was also used. Communication between the UC-Phone and the server was made through PHS circuits. Relationships between the terminals and the respective servers are shown in Fig. 2.

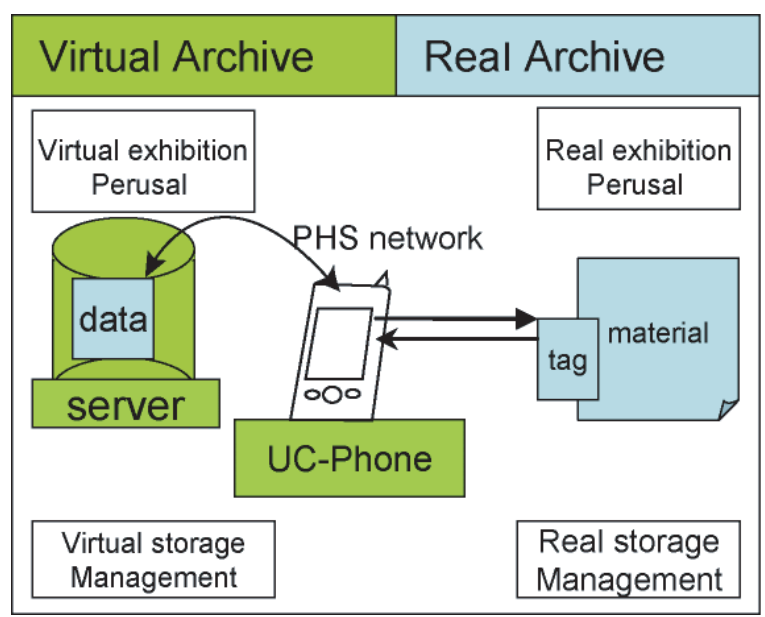

Fig. 2 Network relation in the archive. 


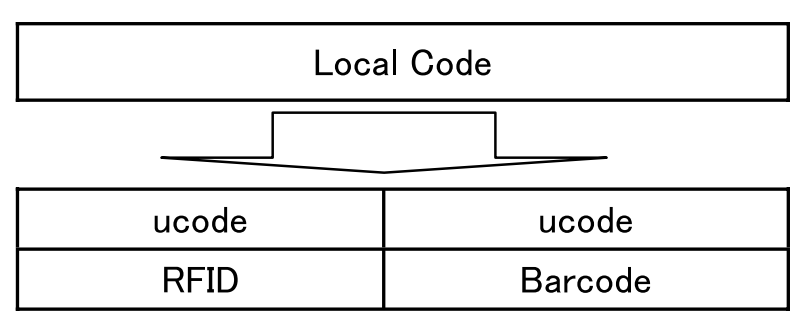

Fig. 3 Relations among the respective codes.

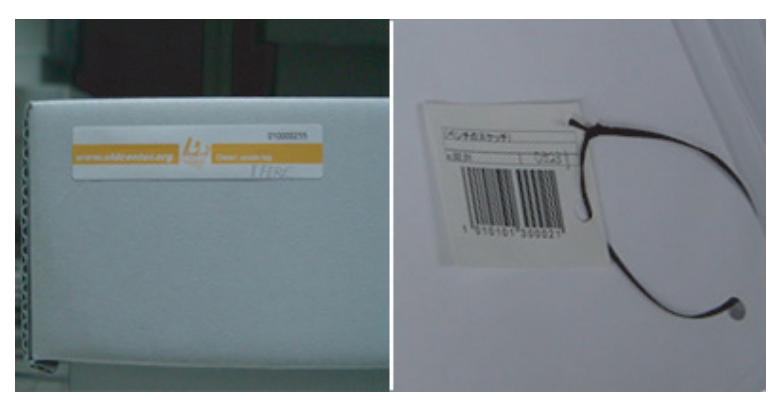

Fig. 4 Barcode and RFID tag assigned to material container.

\subsection{Assignment of IDs}

Various kinds of materials are stored in the real archive, and it is necessary to assign unified IDs to them. When IDs are assigned, ones that can be applied to other materials in the laboratory are provided and assigned. These IDs are not a code to be used for the RFID tags, such as the ucode to be discussed later, but are IDs that can be confirmed visually in local environments. After the local IDs are set, they must correspond to the ucode assigned to the RFID. In the case of the RFID used in this project, the ucode had already been assigned. Although it is possible to configure the ucode to reflect the local ID, the local ID and the ucode were used commonly in the experiment, and the respective IDs correspond to the ucodes as shown in Fig. 3, since the terminal is still in the experimental stage.

To read the ubiquitous terminal from the materials, it is possible to read both the RFID tag and the barcode as shown in Fig. 4. In the database of the virtual archive, all of the codes read from both the RFID tag and the barcode will be handled as "ucodes". Thus, both the RFID and the barcode can be used. Therefore, whether the RFID tag or the barcode should be assigned to the materials was considered based on our study of the differences between them and the characteristics of the materials.

\subsection{RFID and barcodes}

In ubiquitous computing, electronic tags, such as RFID tags, are often used. As an alternative, existing
Table 1 Kinds of codes assigned to the respective materials.

\begin{tabular}{l|l}
\hline Type of material & Type of tag/code \\
\hline Paper materials & Barcode \\
\hline Construction materials & RFID \\
\hline Storage container box & RFID \\
\hline Other media & Barcode \\
\hline
\end{tabular}

barcodes can be provided based on the local environment and can be used inexpensively by using conventional applications. The advantage of the RFID is the fact that various kinds of functions can be considered. For instance, many tags can be read at once by applying advanced code-reader's technologies. Since such codes do not depend on the printed surface, there is no possibility that they will become unreadable due to contamination. In the case of paper materials, barcodes are contained in unified envelopes made of neutral paper, and these envelopes are stored in boxes made of neutral paper. Thus, if barcodes are put in a safe place on the wrapping paper, they are unlikely to deteriorate and will not be accidentally removed. Therefore, it is appropriate to use barcodes and pay thoughtful attention to the ink to be used.

Unlike paper documents, however, it is difficult to store some large, hard materials such as construction materials in unified storage bags. If the barcode is put somewhere on the surface, applying the recognizing device may be difficult, depending on the storage conditions. Moreover, the printed surface of the barcode may be contaminated due to removal or deterioration. Therefore, it is preferable to apply the RFID to such materials as construction materials.

For the above-mentioned reasons, the RFID and the barcode must be used on a case-by-case basis in consideration of their advantages and disadvantages, as well as characteristics of the materials. However, only a limited number of devices are capable of reading both RFID tags and barcodes. If the coding system has been decided in accordance with the specifications of a certain device, operation of the archive may have problems when that device is no longer available. However, the verification experiment in this project aims to collect information to aid decision-making for constructing this system in the future. Thus, we experimentally applied both RFID and barcodes to the archive in order to obtain information for verifying their performances. The RFID and the barcodes correspond to the respective materials as shown in Table 1.

\subsection{Construction of system}

After setting the various kinds of specifications as 
described in the previous section, we constructed the system implementing ubiquitous computing. The procedures for constructing the system are shown in Fig. 4. First, the codes and tags were assigned to the respective materials. Then, the data communication function for connection with PHS terminals was added to the server of the virtual archive so that it was able to receive communication signals via PHS. This allowed the server and the ubiquitous terminal to communicate through PHS circuits. The server was constructed with the Microsoft Internet Information Server and is capable of storing data to be perused from a personal computer, as well as data to be perused from the ubiquitous terminal. The following data are stored in the database: (1) content information, (2) physical information, (3) material history management, (4) completion/incompletion of digital photographing, and (5) relevant material information.

The above-mentioned (1) is basic information about the contents, and (2) is mainly information about physical forms and states of the materials. Category (3) indicates the lending status of the materials over the entire time during which they were stored in the container. Category (4) indicates whether or not digitization of the respective materials has been completed. Category (5) indicates the relevant information about other materials. These kinds of information can be perused, updated, and revised from the management screen (Fig. 5) of the Tsuboi Residence Architectural Documents Archive on the PC.

The ubiquitous terminal recognizes the ID once it reads the RFID tag or barcode. Then it requested the required ID data of the server through PHS communication. The server send the applicable data to the ubiquitous terminal and the terminal display the data. A function to update and revise information from the ubiquitous terminal has not yet been added.

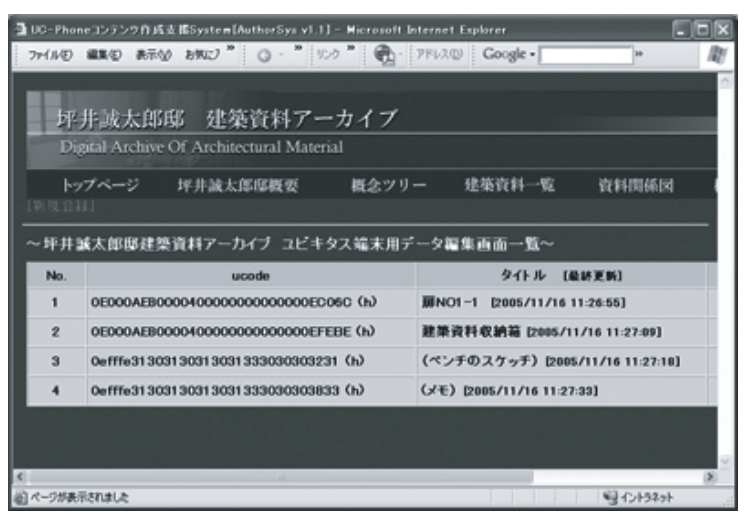

Fig. 5 Reference/Editing management screen of the archive for perusing on a PC.

\section{Operation after constructing archive}

\subsection{Outlines of operation}

The real/virtual archive using the ubiquitous computing was constructed with the contents as mentioned in the previous section. The operating method is shown in Fig. 6. How these contents are operated practically is described in this section.

\subsection{Perusal with $(A)$ virtual archive}

It has become possible to confirm the lending status of the materials within the project and the progress of digitization, as well as searching metadata of the materials on the virtual archive. Thus, both the real archive's status and the contents of the virtual archive can be visually confirmed. The progress for constructing the virtual archive can be confirmed from the real archive. By confirming these kinds of information, the archive conditions of both of the real and virtual archives can be comprehended.

\subsection{Construction and confirmation of updating infor- mation on (B) virtual archive}

In digitization of the materials, parts of the materials that were not yet photographed were digitized the tags were assigned. For the photographed materials, the digitization completion was put into the data on the virtual archive. The ubiquitous terminal can thus be used for selecting materials to be digitized the next time, and the information can be confirmed in the library.

\subsection{Storage/management on (D) real archive}

It is possible to confirm the lending status of the respective materials, the completion, or the incompletion of the digitization within the storage environment where the materials are stored. Titles of the materials are often described on the management containers, and

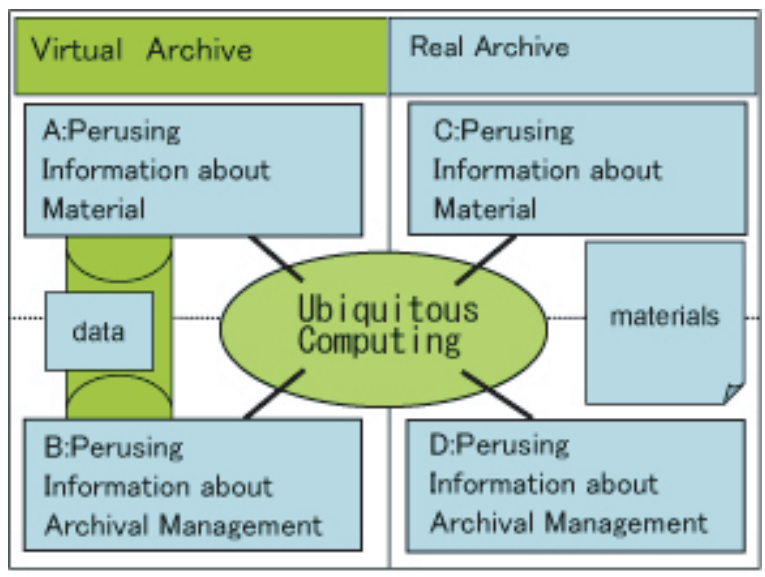

Fig. 6 Roles of the ubiquitous computing in the archive. 


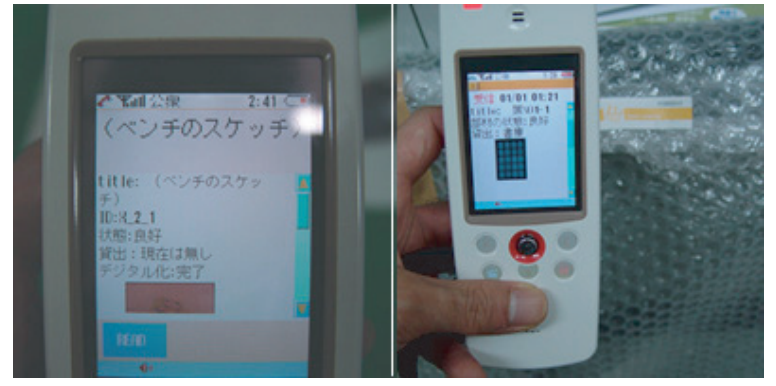

Fig. 7 Reference of the material information with the ubiquitous terminal.

the ubiquitous terminal is not required. However, it is possible to obtain more detailed information and for information to be updated very frequently on site. Although this information can also be confirmed with a PC or a catalog, the required capacity for processing is large, and it is necessary to search for an applicable item from a large number of materials. In the case of the ubiquitous terminal, the required information can be obtained without unnecessary information being obtained as well.

\subsection{Use in constructing virtual archive}

In the processes for constructing the archive, it is possible to confirm the materials by first using the small-sized terminal at the time for picking up the materials in the archive (D). Then, the state of the real archive (D) and the progress of the digitization of (B) can be confirmed so that it is possible to select the materials subject to the next photography session. This process allowed smoother progress in the project.

The above-mentioned descriptions are processes of the verification experiments on the archive. The results and the remaining issues of the experiment currently available are described in the next section.

\section{Results and remaining issues 5.1 Results}

The results of the design and construction of the real/virtual archive using the ubiquitous computing for the Tsuboi Residence Architectural Material Archive are summarized as follows. In this section, common results for the archive are explained separately.

\subsubsection{General results for archive}

1) We verified the basic construction of the archive linking real information and virtual information by using ubiquitous computing.

2) In the real archive, management convenience has been improved and information accumulated.

3) In the virtual archive, information of the real archive can be obtained from the virtual archive by using a lightweight terminal.

4) For the entire archive, it has become possible to share information required for making the flow from (D) to (A) via (B) and returning to (D) again more efficient for constructing the virtual archive.

\subsubsection{Results for architectural archive}

The results for the architectural archive are as follows.

1) Since the construction materials that need to be stored are of various kinds of shapes, such as plain and solid articles, they tend to be distributed to several locations for storage. However, it has become possible to use information about the materials stored in different locations.

2) Shapes of the construction materials are diverse and printing characters and other marks on them may be difficult. Even in such cases, the IDs of the materials can be managed with tags.

The above-mentioned results can be considered current. However, various kinds of issues remain; these are discussed in the next section.

\subsection{Remaining issues}

\subsubsection{Operational issues}

A function for updating the history management data from the ubiquitous terminal to the server on the virtual archive is not currently available, and information can be perused or confirmed only on a PC. Providing a function by which the ubiquitous terminal could correct and update the data in the future would be preferable.

In the network environment, situations exist in which establishing communication links is difficult due to radio wave environments, since a PHS terminal is used in the system. Thus, results take about 10 seconds to display, and the speed for the user is very slow. Therefore, it is necessary to set up a network environment as well as a wireless LAN environment.

Furthermore, since the verification coverage is limited to the archive, its opportunities for use are still few. We think that further verification must be executed for the purpose of expanding the applicable range to include the materials that will be used more frequently in the future.

\subsubsection{Perusal with (C) real archive}

As mentioned, this project has no place or space for exhibition and perusal for the public (C). Thus, it is currently impossible to use ubiquitous computing for perusing and exhibiting the materials. However, it is possible to exhibit the materials at another archive and use the information about the details of the materials from a remote location by using the ubiquitous termi- 
nal. Thus, we think that it is necessary to use ubiquitous computing for the entire system, including Phase (C) as well as Phases (A), (B), and (D), which were examined in this research.

\subsubsection{RFID and barcodes}

The RFID and the barcode were introduced experimentally in this project. The barcode seems sufficient for use in management in which the materials are confirmed one by one. Meanwhile, we found that the RFID is extremely efficient for large-sized materials, materials that can be contaminated easily, and storage environments where printed documents may be deteriorated.

Many cultural materials, such as statues and attachments of buildings, are mounted or installed outdoors. For the ID management of these kinds of materials, the RFID is effective for setting a tag approximate to the material instead of the material itself. We think the technology for the reading device will evolve in the future, performance will improve, and superior functions for barcodes will be added. It is expected, in particular, that some superior conveniences will be provided in the future, for instance the ability to recognize many materials at once and the inclusion of autonomous functions. Thus, the benefit of using RFID seems to expand depending on the purpose of use and the subject materials for the archive.

As already mentioned, both the RFID and the barcode have advantages, and further study must be conducted through experiments and verification. After such study, we need to examine the possibility of unifying the method or using both methods, considering the relationship between the materials and tags/codes and the technical development of ubiquitous computing in the future.

This project was applied only to the architectural materials of the Tsuboi family. In order to adapt this system to all materials generally, further verification activities like this must be conducted.

\subsubsection{Assignment of IDs to cultural resources}

As for the IDs for this project, the ucodes assigned by the Ubiquitous ID Center were used in the local environment, the ucodes were unique IDs assigned to the applicable items, and these will not be duplicated with other IDs issued by the Ubiquitous ID Center. If the range of use of the IDs is limited within the respective facilities, creating IDs based on the local system is sufficient. However, when the materials are lent to other facilities in the future, using codes assigned by the Ubiquitous ID Center is expected to improve the convenience for management. Since the subjects for assigning the IDs are public assets, such as cultural re- sources, it is preferable to apply a neutral coding system without any interests. Thus, we think that further discussions and studies on this issue are necessary.

\subsubsection{Conclusion and future prospects}

We completed the experiment for the Tsuboi Residence Architectural Material Archive. It is important to link the real archive and the virtual archive, and we found that further verifications, studies, and discussions based on the results of this research are required in the future for materializing this link.

\section{References}

[1] N. Togiya, T. Soeno, S. Yamane, and A. Baba, "Design and Construction of Real/Virtual Archive Using Ubiquitous Computing", In Proceedings of Computers and Humanities 2005, IPSJ, pp. 79-86, 2005.

[2] F. Khan, "Museum Puts Tags on Stuffed Birds", RFID Journal, pp. 27-30, 2004.

[3] F. Kusunoki, "Hakubutsukan ni okeru kagaku kyouiku shien system no kaihatsu to Hyouka no kenkyu", "Research for Development and Evaluation of the Science Instruction Support System in the Museum", Report of Grant-in-Aid for Scientific Research, 2003 (in Japanese).

[4] H. Hashizume, "Digital museum ni okeru sankagata souzougakushu system", "The participated type creation learning system in a digital museum", Report of Grantin-Aid for Scientific Research, 2002 (in Japanese).

[5] E. Yamazaki, "RFID tag no IT toshokan eno ouyou", "Applying RFID tags to IT library", The Journal of Information Science and Technology Association, vol. 52, no. 12, pp. 609-614, 2002 (in Japanese).

[6] N. Togiya and A. Baba, "Practice and verification of providing metadata to modern private material", In Proceedings of Computers and Humanities IPSJ, Tokyo, 2004, 99-98, 2004.

[7] N. Togiya, M. Fujihara, and A. Baba, "Constructing and Role of the Ontology for Creating Digital Archive of Architecture of Architectural Material", Journal of Japan Society of Information and Knowledge, vol. 15, no. 2, pp. 19-24, 2005.

[8] http://www.tengine4u.com/ucphone/welcome.htm (Visited March 31, 2006)

[9] http://www.ubin.jp/

[10] http://www.uidcenter.org/ 


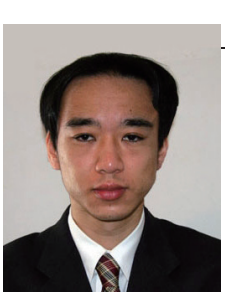

\section{Norio TOGIYA}

Doctoral course student at the Graduate School of Interdisciplinary Information Studies, University of Tokyo. Fields of specialization are archive and museum informatics.

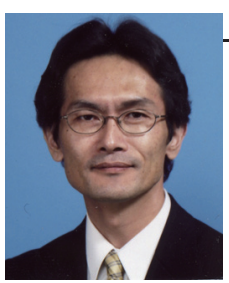

\section{Akira BABA}

Professor in the Interfaculty Initiative in Information Studies, University of Tokyo. Studied in the doctoral program of the Graduate School of Waseda University. Fields of specialization are early modern Japanese economic history, historical informatics, archive sciences, and game studies. 